\title{
Modelo de associação de molas em paralelo em atividades de ensino de Física: uma análise do domínio de validade
}

Model of association of springs in parallel in activities of teaching of Physics: an analysis of the domain of validity.

\author{
Ricardo Robinson Campomanes ${ }^{*}\left[\right.$, Leonardo Albuquerque Heidemann ${ }^{2}$, Eliane Angela Veit ${ }^{2}[$ \\ ${ }^{1}$ Universidade Federal de Mato Grosso, Instituto de Ciências Naturais, Humanas e Sociais, Sinop, MT, Brasil \\ ${ }^{2}$ Universidade Federal do Rio Grande do Sul, Instituto de Física, Porto Alegre, RS, Brasil
}

Recebido em dia de 03 de abril de 2019. Revisado em 20 de setembro de 2019. Aceito em 11 de novembro de 2019

\begin{abstract}
Associações de molas são frequentemente exploradas no ensino de Física por meio de um modelo teórico que define a constante elástica equivalente de um conjunto de molas em paralelo como o somatório das constantes elásticas de cada uma das molas associadas. Como qualquer modelo científico, essa representação é construída assumindo uma série de idealizações que delimitam o seu domínio de validade. No referido modelo, pressupõe-se que, quando uma força é aplicada, as molas associadas distendem-se igualmente, ou seja, apresentam uma mesma deformação. Para o caso em que duas molas são usadas para sustentar um objeto verticalmente, dificilmente teremos essa condição respeitada. No presente artigo, apresentamos uma discussão sobre a associação de duas molas em paralelo sob a perspectiva do processo de modelagem segundo Bunge. Propomos uma expansão do modelo teórico tradicionalmente explorado considerando a associação de duas molas em paralelo que não se deformam igualmente. Apresentamos também uma contrastação empírica do modelo tradicional e do modelo expandido proposto. Entendemos que a discussão construída tem potencial para, além de proporcionar a mobilização de conhecimentos de Física básica, fomentar discussões sobre o papel representacional do conhecimento científico, possibilitando situações que demandam reflexões sobre a natureza da Ciência.
\end{abstract}

Palavras-chave: Lei de Hooke, constante elástica, associação de molas em paralelo, modelo científico.

Spring associations are often explored in Physics teaching through a theoretical model that defines the equivalent elastic constant of a set of springs in parallel as the sum of the spring constants of each of the associated springs. Like any scientific model, this representation is constructed by assuming a series of idealizations that delimit its validity domain. In the mentioned model, it is assumed that, when a force is applied, the associated springs extend equally, that is, they present a same deformation. For the case where two springs are used to sustain an object vertically, we will hardly have this condition respected In this paper, we present a discussion about the association of two springs in parallel from the perspective of the Bunge modeling process. We propose an expansion of the traditionally explored theoretical model considering the association of two parallel springs that do not deform equally. We also present an empirical comparison of the traditional model and the proposed expanded model. We understand that the discussion built has the potential to, besides providing the mobilization of basic physics knowledge, foster discussions about the representational role of scientific knowledge, enabling situations that demand reflection on the nature of Science.

Keywords: Hooke's law, spring constant, parallel springs, scientific model.

\section{Introdução}

Vincular teorias e realidade é, sem dúvida, um dos desafios enfrentados pelos professores de Física. Os livros didáticos muitas vezes tratam de situações físicas previamente idealizadas, não explicando as relações entre os conhecimentos abordados e o mundo vivencial do aluno [1]. Por outro lado, a literatura da área mostra que é comum os estudantes entenderem que modelos científicos são cópias fiéis da realidade $[2,3]$. Uma alternativa promissora para proporcionar aos alunos uma reflexão crítica sobre as relações entre teorias e realidade nos cam-

*Endereço de correspondência: ricardo_speru@yahoo.com.br pos da Física, evidenciando o caráter representacional do conhecimento científico, é o enfoque na modelagem científica [4-7]. Nessa perspectiva, os modelos são entendidos como representações simplificadas de eventos reais, mediando as relações entre teoria e realidade. Seguiremos a abordagem da modelagem científica segundo o físicomatemático e filósofo da ciência Mario Bunge [8,9]. Ele menciona na sua obra Teoria e Realidade (1974, p. 31) [10]:

Preocupar-nos-emos com objetos-modelo e modelos teóricos como esboços hipotéticos de coisas e fatos supostamente reais. Assim um 
fluido pode ser modelado como um contínuo dotado de certas propriedades e semelhante objeto-modelo pode ser enxertado em uma das várias teorias gerais, digamos a mecânica clássica ou mecânica relativística geral. Do mesmo modo é possível modelar um organismo de aprendizagem como uma caixa negra equipada com determinados terminais de entrada e saída e pode-se desenvolver este objeto-modelo em um sistema dedutivo hipotético. Em qualquer dos casos produz-se uma teoria específica ou modelo teórico de um objeto concreto. $\mathrm{O}$ que se pode submeter a provas empíricas são tais modelos teóricos: as teorias gerais despreocupadas com particulares permanecem incomprováveis, a menos que sejam enriquecidas com modelos de seus referentes. E os objetos-modelo mantêm-se estéreis a não ser que sejam introduzidos ou desenvolvidos em alguma teoria.

Neste artigo, abordamos um problema de Física sob a perspectiva da modelagem científica, destacando que os modelos científicos, em função das idealizações consideradas, possuem domínios de validade que podem ser expandidos por meio de modificações em suas hipóteses. Especificamente, abordamos as limitações do modelo de associação de duas molas em paralelo tradicionalmente abordado nos livros didáticos de Física [11, 12]. Destacamos como modificações em suas hipóteses possibilitam uma expansão do seu domínio de validade. Adotamos as concepções epistemológicas assumidas na Modelagem Didático-Científica. Desse modo, entendemos que um modelo teórico constitui um sistema hipotético-dedutivo específico que representa objetos ou eventos reais (ou supostos como tais) particulares fundamentados em teorias gerais da Ciência. Por exemplo, um modelo de gás ideal, fundamentado na Mecânica Estatística Clássica, pode ser usado, com algum fim específico, para representar um gás real confinado. São também exemplos de modelos teóricos da Física o modelo newtoniano de pêndulo simples, o modelo de fluido ideal, e o modelo atômico de Bohr. Não é nosso objetivo aqui, no entanto, expor pormenores dessas concepções, que podem ser consultadas em Heidemann, Araujo e Veit [1].

Nosso objetivo é apresentar uma experimentação explicitando como o processo de modelagem orienta a construção do conhecimento científico, e evidenciando como tal processo possibilita uma melhor compreensão sobre as potencialidades e limitações dos modelos científicos. Tal exemplo pode ser explorado por professores de Física para a abordagem de conceitos importantes da Mecânica, como a Lei de Hooke, de forma integrada com debates sobre epistemologia na Física. Nesses debates, podem ser discutidas questões sobre, por exemplo, como o conhecimento científico é construído, sobre os objetivos dessa construção, e sobre as potencialidades e limitações desse conhecimento, proporcionando aos alunos a oportuni- dade para o desenvolvimento de criticidade sobre temas centrais em reflexões sobre a natureza da Ciência.

Robert Hooke, cientista inglês contemporâneo de Isacc Newton, dedicou-se a pesquisas em diferentes áreas, realizando avanços na física e na biologia. Uma de suas principais descobertas, que lhe deu maior notoriedade, é a relação observada entre a força aplicada em uma mola e a deformação produzida, hoje conhecida como a Lei de Hooke. Já em 1675, Hooke escrevia em seu diário: "todas as molas em liberdade dobram espaços iguais por igual aumento de peso' ${ }^{\prime}[13]$.

É importante destacar que, ainda que nos restrinjamos neste artigo a discutir situações em que a relação entre a força aplicada e a deformação sofrida pelas molas é linear, elasticidade não implica proporcionalidade linear. Um material é elástico quando não sofre deformações irreversíveis [14]; ou seja, ao ser deformado por uma força, volta à sua forma original quando essa força cessa. Molas de maneira geral são elásticas, mas não necessariamente lineares [15]. Somente para elongações da mola em que a distribuição espacial dos elos se torna aproximadamente uniforme, os espaçamentos entre as espiras podem ser considerados iguais e espera-se que a Lei de Hooke seja válida $[16,17]$. Neste artigo usamos molas cilíndricas e nos concentramos na região em que a Lei de Hooke é satisfeita, dentro de certo domínio de validade e grau de aproximação.

No que segue, apresentamos o modelo tradicional de associação de molas em paralelo, que assume que as duas molas se distendem igualmente, e o contrastamos experimentalmente para o caso em que as duas molas têm constantes elásticas semelhantes. Então, propomos uma expansão desse modelo para representar satisfatoriamente situações em que as distensões das duas molas não são necessariamente iguais, ou seja, que extrapolam o domínio de validade do modelo tradicional. O modelo expandido é contrastado empiricamente nos casos em que as constante elásticas são (e não) semelhantes. Entendemos que essa discussão pode ser frutífera para o ensino de Física tanto para a abordagem de conteúdos científicos, como para discussões sobre a natureza da Ciência, pois, assim como diversos autores da área de ensino de Ciência [18-20], assumimos que, enfatizando o papel dos modelos na construção da Física, possibilitamos que os estudantes compreendam o caráter representacional do conhecimento e as consequências dessa característica indelével da Ciência.

\section{O modelo de associação de molas em paralelo nos livros didáticos}

As propriedades elásticas dos materiais são amplamente pesquisadas. Peças de usinagem para equipamentos, amortecedores de veículos, colchões de espuma flexível ou

1 The Diary of Robert Hooke: 1672-1680, ed. Henry W. Robinson and Walter Adams (London: Taylor and Francis, 1935), apud Moyer, p. 267 (tradução nossa) [13]. 
de molas são exemplos de aparatos com delineamento tecnológico fundamentado em propriedades elásticas de materiais. As propriedades elásticas dos materiais são tradicionalmente abordadas no ensino de Física básica com os estudos sobre o comportamento de molas a partir da denominada Lei de Hooke [21, 22]. Tal lei relaciona a força restauradora da mola com a sua deformação da seguinte forma [23, p. 115]:

$$
\vec{F}=-k \vec{x}
$$

sendo $k$ a constante elástica ou de rigidez da mola, $\vec{x}$, a deformação ou a elongação da mola, ou seja, é o deslocamento da extremidade livre da mola em relação à posição em que a mola não está nem comprimida nem distendida $(x=0)$.

Neste ponto, podemos destacar um aspecto básico da natureza do conhecimento científico: o caráter representacional dos modelos teóricos. Assumir que a força elástica em uma mola respeita a Lei de Hooke é uma simplificação da realidade. Tal idealização, no entanto, é satisfatória para se representar o comportamento de molas em diversos casos. Tomando a Lei de Hooke e fundamentando-se na Mecânica Newtoniana, diversos livros didáticos, tanto de nível médio como superior [11, 12][23, p. 133][24], expõem a construção de um modelo teórico para representar associações de molas em paralelo ou adotam esse modelo, sem construí-lo com base nas suas hipóteses básicas. Tal interesse pode ser justificado em função dos diversos sistemas reais que demandam o uso de molas acopladas, como as molas do sistema de amortecimento de motocicletas e automóveis, de equipamentos de Pilates [25], entre outros. Assim, sua abordagem no ensino de Física pode ser problematizada com eventos vivenciais, na qual são considerados os conhecimentos prévios dos estudantes, fundamentada conceitualmente, e validada experimentalmente.

No que segue, detalharemos esse modelo, que denominaremos aqui de Modelo Tradicional para Associações de Molas em Paralelo (MTAMP). A Figura 1 ilustra o objeto-modelo, em que duas molas de mesmo comprimento são associadas em paralelo com uma haste as unindo nas suas extremidades inferiores com a carga suspensa no centro da haste.

Admite-se que:

- as massas das molas e da haste, o empuxo do ar e as pequenas vibrações das molas são desprezíveis;
- para cada uma das molas, a força elástica exercida pela mola (individualmente) é linearmente proporcional à sua elongação, ou seja, $F_{1}=k_{1} x_{1}$ e $F_{2}=k_{2} x_{2}$.

- as molas 1 e 2 e a mola equivalente sofrem iguais elongações, isto é, $x_{1}=x_{2}=x_{E}$.

Pretende-se descrever o comportamento elástico dessa associação de molas em paralelo por uma única mola, denominada de mola equivalente, cuja elongação $x_{E}$ é a mesma das molas 1 e 2 .

Um professor, ao explorar esse modelo teórico, deveria questionar o que acontece quando as constantes elásticas não são aproximadamente iguais. Se há diferença significativa entre as constantes elásticas, necessariamente as forças nas duas molas não podem ser consideradas iguais, ou seja, a força aplicada na haste não é distribuída igualmente nas duas molas. Para a haste permanecer na posição horizontal, é necessário que a carga seja deslocada do centro da haste, como vamos discutir.

Para $k_{1}=k_{2}$, a Figura 2 expõe o diagrama de corpo livre da haste do objeto-modelo da Figura 1. As forças $\vec{F}_{1}$ e $\vec{F}_{2}$ representam as forças restauradoras das molas 1 e 2 , respectivamente, e $\vec{F}$ a força aplicada pela carga suspensa na haste, responsável pela deformação das molas. Assumindo a primeira condição de equilíbrio, temos:

$$
\vec{F}_{1}+\vec{F}_{2}+\vec{F}=\overrightarrow{0}
$$

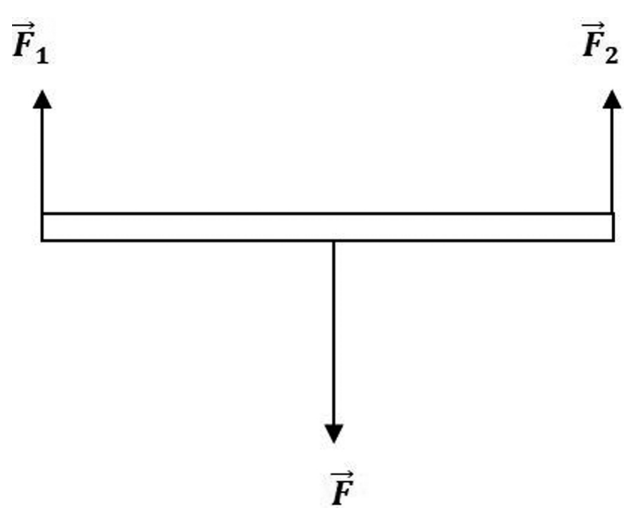

Figura 2: Diagrama de corpo livre da haste. No desenho $F_{1}=F_{2}$ e a carga está pendurada no centro da haste. Se as elongações das molas são iguais $\left(x_{1}=x_{2}\right), \mathrm{k}_{1}$ deve ser igual a $\mathrm{k}_{2}$. Se $k_{1}$ é diferente de $k_{2}$ e $x_{1}=x_{2}, F_{1}$ não pode ser igual a $F_{2}$, como mostrado no desenho, e a carga teria que ser deslocada do centro da haste para manter $\mathrm{x}_{1}=\mathrm{x}_{2}$.

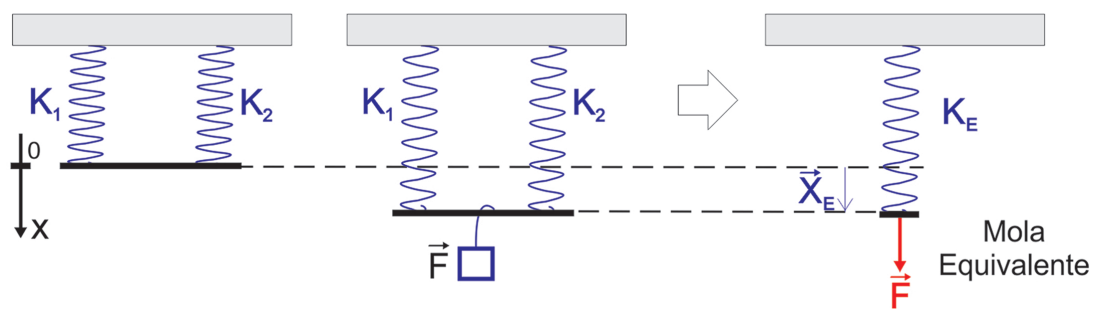

Figura 1: Representação do modelo tradicional para associação de molas em paralelo. 
Usando o módulo dessas forças, temos:

$$
F_{1}+F_{2}=F .
$$

Como assumimos que $\mathrm{F}_{1}$ e $\mathrm{F}_{2}$ obedecem a Lei de Hooke, e que $F_{E}(=F)$ é igual ao módulo da força elástica exercida pela mola equivalente,temos:

$$
k_{1} x_{1}+k_{2} x_{2}=k_{E} x_{E}
$$

sendo $k_{1}$ e $k_{2}$ as constantes elásticas das duas molas isoladas, $k_{E}$, a constante elástica da mola equivalente no modelo tradicional e $x_{E}$ a correspondente elongação equivalente. Usando a hipótese $x_{1}=x_{2}=x_{E}$ na equação (2b), temos:

$$
k_{1}+k_{2}=k_{E}
$$

ou seja, no MTAMP, a constante elástica $k_{E}$ é dada pelo somatório das constantes elásticas das molas isoladas.

Nesta dedução, admitiu-se que as constantes elásticas das duas molas fossem iguais. Caso haja diferença significativa entre as constantes elásticas das molas, $k_{1} \neq k_{2}$, a condição $x_{1}=x_{2}$ não seria satisfeita com a carga sendo colocada no centro da haste. A equação (3) só poderia ser aplicada para $k_{1} \neq k_{2}$ se a carga fosse deslocada do centro, de modo que as elongações de ambas as molas fossem iguais. Esse aspecto não costuma ser discutido nos livros didáticos, mas vamos discuti-lo detalhadamente na próxima seção.

Um professor que explora esse modelo teórico pode, portanto, conduzir debates sobre as implicações das idealizações consideradas em um modelo no seu grau de precisão, ressaltando aspectos da construção do conhecimento científico.

Para contrastar empiricamente o MTAMP, coletamos dados experimentais. Para isto, previamente foram determinadas experimentalmente as constantes elásticas de duas molas $\left(\mathrm{k}_{1}\right.$ e $\left.\mathrm{k}_{2}\right)$, medindo, para diversos valores de força aplicada pela carga suspensa na mola, a elongação da mola quando o conjunto corpo suspenso e mola atingem o repouso. Com base na curva de ajuste desses dados, obtivemos a constante elástica de cada uma das molas.

Posteriormente, as duas molas de mesmo comprimento foram suspensas em um suporte e conectadas em seu extremo inferior por uma fina haste de metal de alumínio. As molas ficaram, então, associadas em paralelo, como mostra a Figura 3. No centro dessa haste foi pendurada uma carga (Figura 3.a e 3.c). Foram utilizados como carga arruelas de massas de 10 gramas e 20 gramas, inseridas num gancho de 10 gramas. Os instrumentos utilizados para as medições, e respectivas incertezas, foram: i) de massa, uma balança de pratos de 0,05 grama, e ii) de comprimento, uma régua fixa na vertical, de 0,0005 metro. Foi usado como valor da aceleração da gravidade $\mathrm{g}=9,795 \pm 0,003 \mathrm{~m} / \mathrm{s}^{2} \mathrm{~L}^{2}$

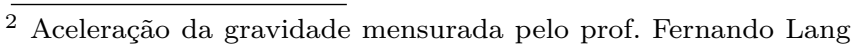
da Silveira no mesmo local onde os experimentos relatados neste

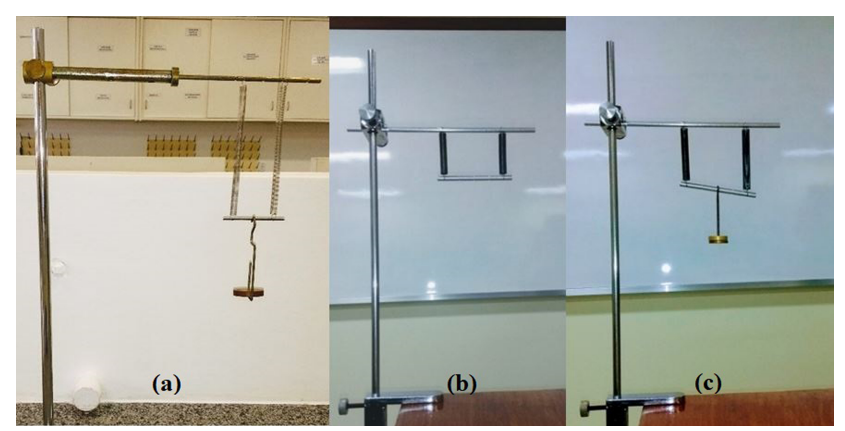

Figura 3: a) Se $k_{1} \approx k_{2}$, a haste permanece horizontal com carga suspensa. Se $k_{1} \neq k_{2}$, em (b) a haste fica na posição horizontal quando não há carga suspensa, e em (c) se inclina quando há carga.

A situação exposta na Figura 3.a respeita aproximadamente as idealizações adotadas no MTAMP, em que se considera iguais deformações das molas 1 e 2, para valores de $k_{1} \approx k_{2}$. No entanto, tal modelo não representa satisfatoriamente a configuração experimental exposta na Figura 3.c, para molas com constantes elásticas $k_{1} \neq k_{2}$ com a carga suspensa fazendo com que a haste não fique na posição horizontal, apesar de que estivesse nessa posição sem a carga suspensa (Figura 3.b). Logo, não é adequado assumir a hipótese de igualdade das elongações das molas, ou seja, que $x_{1}=x_{2}=x_{E}$, e é necessário expandir o MTAMP, determinando a constante elástica equivalente dessa configuração, conforme será debatido na Seção 3. Tal fato, para o docente interessado em fomentar debates sobre a natureza da Ciência, fornece uma situação que exemplifica como procedimentos de controle de variáveis são centrais em experimentos em que se pretende contrastar empiricamente modelos teóricos da Física, e como esses procedimentos são dirigidos pelas idealizações consideradas no modelo contrastado. Sobre isso, Bunge (1974, p. 24) [10] sugere:

Se o modelo teórico $\mathrm{T}_{s}$ não concorda com os fatos e se for possível estar razoavelmente seguro de que isto não se deve ao erro dos dados experimentais, será preciso modificar as ideias teóricas. Isto é mais rápido de se dizer do que se fazer, pois há diversas possibilidades: pode-se querer variar o objeto-modelo $\mathrm{m}$, querer guardá-lo e adotar uma outra teoria geral $\mathrm{T}_{g}$, pois toda teoria especial é constituída, em princípio, de um $\mathrm{m}$ e de uma $\mathrm{T}_{g}$ que não se deixam determinar reciprocamente (. ..). O tipo de mudança preconizada dependerá dos serviços prestados no passado pelo objeto-modelo e pelas teorias gerais envolvidas. Se estas últimas foram bem sucedidas antes, será prudente tentar um novo objeto-

artigo foram realizados. Detalhe sobre os procedimentos dessas medições estão disponíveis em https://www.if.ufrgs.br/ lang/Textos/GRAVIDADE.pdf 
modelo; para isso, ter-se-á talvez necessidade de novos dados empíricos.

Voltando à configuração experimental referente ao caso $k_{1} \approx k_{2}$ (Figura 3.a), os dados empíricos coletados dessa configuração de molas em paralelo, como também das molas por separado, são plotados na Figura 4, onde se vê, além disso, a curva de ajuste para a determinação da constante elástica de cada uma das molas, assim como da associação das molas em paralelo. A incerteza na medida da elongação equivalente, considerando o efeito de paralaxe, foi de 0,002 metro. O ajuste linear dos dados foi realizado usando o software Origin, o qual fornece tanto o coeficiente angular quanto seu desvio padrão, ou seja, o valor de constante elástica e a sua incerteza, resultando $k_{1}=19,2 \pm 0,4 \mathrm{~N} / \mathrm{m} ; k_{2}=19,8 \pm 0,4 \mathrm{~N} / \mathrm{m}$ e o valor empírico da constante para as molas associadas em paralelo é dada por $k_{\text {exp }}=(38,3 \pm 0,9) \mathrm{N} / \mathrm{m}$. Por outro lado, pode-se predizer a partir do MTAMP o valor da constante elástica equivalente, dada pela soma das constantes elásticas das duas molas $k_{E}=(39,0 \pm 0,6)$ $\mathrm{N} / \mathrm{m}$.

Considerando tanto as incertezas da predição do modelo teórico quanto da medida realizada, o valor estimado $\left(\mathrm{k}_{\mathrm{E}}\right)$ e o valor experimental $\left(\mathrm{k}_{\mathrm{exp}}\right)$ para a constante elástica equivalente se sobrepõem. Pode-se, portanto, afirmar que os dados experimentais coletadas dão apoio empírico ao MTAMP, corroborando sua validade para situações em que as constantes elásticas das molas acopladas são similares $\left(k_{1} \approx k_{2}\right)$. Ou seja, é validada a hipótese de que ambas as molas 1, 2 e a mola equivalente apresentam as mesmas elongações. No entanto, como já mencionamos, tal hipótese não é respeitada na situação exposta na Figura 3.c. Para representarmos satisfatoriamente tal situação, expandimos o MTAMP e o contrastamos empi-

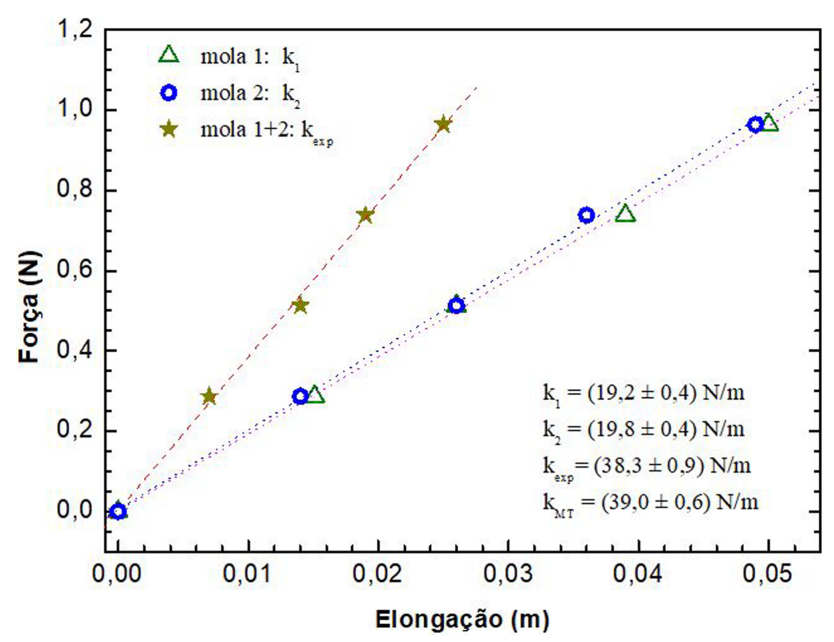

Figura 4: Força aplicada em função da elongação produzida em cada uma das molas, com constantes elásticas $\mathrm{k}_{1} \approx \mathrm{k}_{2}$, e na associação de molas, com a carga aplicada no centro da haste. $O$ valor da constante elástica predita pelo MTAMP é representado por $\mathrm{k}_{\mathrm{MT}}$. ricamente. Os procedimentos realizados são expostos na próxima seção.

\section{Uma expansão do modelo de associação de molas exposto em livros didáticos}

A discussão proposta nessa seção se constitui em um exemplo sobre como os modelos teóricos podem ser expandidos por meio de modificações nas suas hipóteses mais básicas, ampliando seus domínios de validade. Desse modo, pode fomentar debates em aulas de Física sobre o crescimento em superfície do conhecimento científico, que é característico do trabalho dos cientistas em períodos de Ciência normal.

Para explicar a configuração experimental da Figura 3.c, construímos uma Expansão do MTAMP (EMTAMP) para predizer o valor da constante elástica equivalente de duas molas, com diferentes constantes $k_{1}$ e $k_{2}$, associadas em paralelo. A Figura 5.b ilustra a representação do objeto-modelo investigado na EMTAMP, em que duas molas inicialmente de mesmo comprimento são associadas em paralelo com uma haste as unindo nas suas extremidades inferiores (ver Figura 5.a). Cabe destacar que a expansão do modelo não pressupõe que a carga seja aplicada no centro da haste. A carga é pendurada a uma distancia $L_{1}$ do extremo inferior da mola 1 , sendo que a distância entre as extremidades inferiores das molas é $\mathrm{L}$ (= $L_{1}+L_{2}$, comprimento da haste).

Nesse modelo teórico, busca-se descrever o comportamento elástico de uma associação de molas em paralelo, unidas por uma haste horizontal com uma carga suspensa em um determinado ponto da haste, por uma única mola, denominada de mola equivalente ${ }^{3}$ (ver Figura 5.c). Impõe-se que a mola equivalente, quando sujeita à carga $\vec{F}$, tenha elongação $x_{E}$.

Assume-se que:

- as massas das molas e da haste, o empuxo do ar e as pequenas vibrações das molas são desprezíveis;

- para cada uma das molas, a força elástica exercida pela mola (individualmente) é linearmente proporcional à sua elongação, ou seja, $F_{1}=k_{1} x_{1}$ e $F_{2}=k_{2} x_{2}$;

- a elongação da mola equivalente $\vec{x}_{E}$ é dada pelo deslocamento entre a posição inicial da haste sem carga (posição $x=0$, linha horizontal tracejada da figura 5) e o ponto de suspensão da carga pendurada na haste quando as molas estão deformadas (ver seta pontilhada de cor vermelho da Figura 5.b);

- as duas molas estão paralelas.

A elongação equivalente do sistema, $\vec{x}_{E}$, não somente está relacionada com as elongações das molas 1 e 2, mas com os valores de comprimentos $L_{1}$ e $L_{2}$ da haste. Ao se deslocar a haste da posição inicial horizontal $(x=0$, sem

\footnotetext{
3 Também neste caso, na mola equivalente é aplicada a mesma força F que é aplicada no conjunto de molas. A elongação equivalente se relaciona às elongações $x_{1}, x_{2}$ e $L_{1}$, e sua energia potencial elástica é a soma das energias potenciais elásticas das molas 1 e 2.
} 


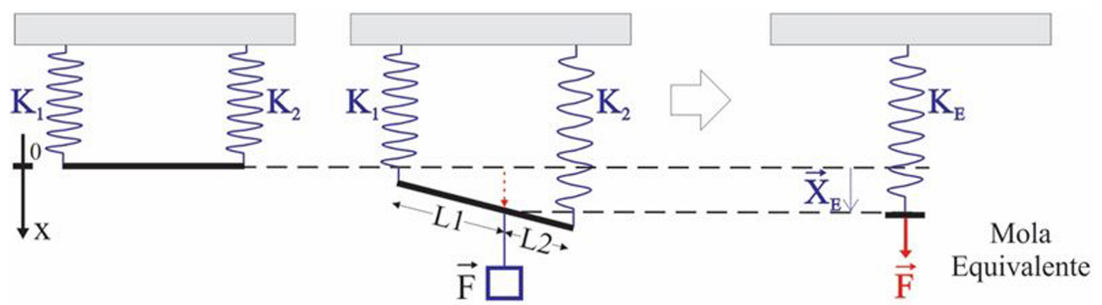

( a )

(b)

(c)

Figura 5: Representação da expansão do modelo tradicional para associação de molas em paralelo.

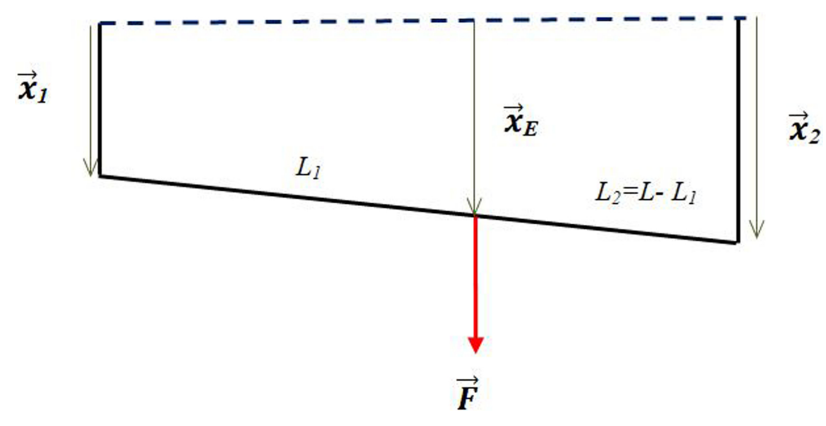

Figura 6: A linha tracejada indica a posição inicial da haste, sem carga. Com carga, a haste desce, saindo da posição horizontal. Pode-se ver na figura um trapézio grande, que contém dois menores. Observe-se que $\vec{x}_{E}$ é o deslocamento da posição inicial da haste até a posição final da mesma haste no ponto onde é aplicada a força $\vec{F}$.

carga) até uma posição final inclinada, as molas e a haste formam um trapézio (ver Figura 6). A propriedade de um trapézio é que suas bases são paralelas, ou seja, $\vec{x}_{1} / / \vec{x}_{2}$, por conseguinte, paralelo a $\vec{x}_{E}$. Nessa figura observamse dois trapézios menores semelhantes e pode-se, então, escrever as seguintes as proporções:

$$
\begin{aligned}
& \frac{x_{1}}{L_{1}}=\frac{x_{E}}{L_{2}} \rightarrow x_{E} L_{1}=x_{1} L_{2} \\
& \frac{x_{2}}{L_{2}}=\frac{x_{E}}{L_{1}} \rightarrow x_{E} L_{2}=x_{2} L_{1}
\end{aligned}
$$

Somando 4a $+4 \mathrm{~b}$, temos: $x_{E}\left(L_{1}+L_{2}\right)=x_{1} L_{2}+$ $x_{2} L_{1}$.

Como $L=L_{1}+L_{2}$, tem-se:

$$
x_{E}=\frac{x_{1} L_{2}+x_{2} L_{1}}{L} .
$$

A expressão $4 \mathrm{c}$ é a condição de paralelismo, a hipótese principal do modelo teórico EMTAMP (diferente da hipótese da igualdade das elongações do modelo tradicional, MTAMP)

A Figura 7 apresenta o diagrama de corpo livre da haste do objeto-modelo da Figura 5.b. Identificamos as forças exercidas pelas molas sobre a haste como $\vec{F}_{1}$ e $\vec{F}_{2}$, e a força deformadora exercida pelos corpos pendurados em um ponto qualquer da haste como $\vec{F}$.

Como a haste não se movimenta, então ela se encontra em equilíbrio tanto de translação quanto de rotação.

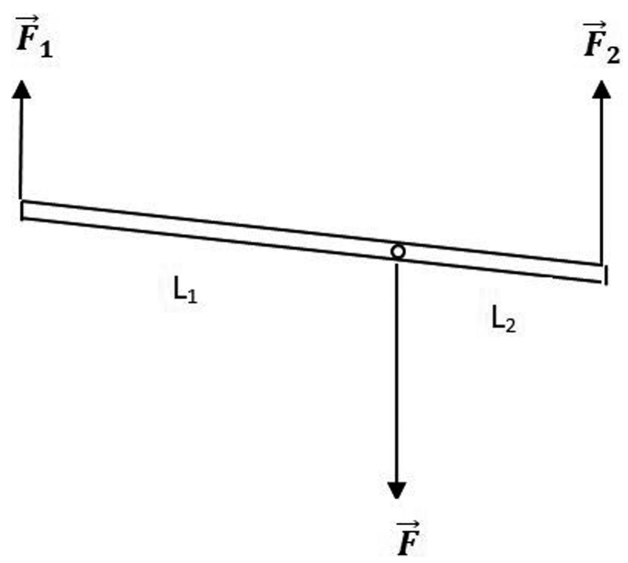

Figura 7: Diagrama de corpo livre da haste do objeto-modelo na EMTAMP.

Logo, da Primeira Condição de Equilíbrio (equilíbrio de translação), temos que os módulos das forças:

$$
F_{1}+F_{2}=F .
$$

Assumindo que $\mathrm{F}_{1}, \mathrm{~F}_{2}$ e $F_{E}(=F)$ obedecem a Lei de Hooke, da equação (1) temos:

$$
F_{1}=k_{1} x_{1}, F_{2}=k_{2} x_{2} \text { e } F=k_{E} x_{E} .
$$

sendo $k_{1}, k_{2}$ e $k_{E}$ as constantes elásticas dessas molas e $x_{1}, x_{2}$ e $x_{E}$, as respectivas elongações.

Isolando-se $k_{E}$, tem-se:

$$
k_{E}=\frac{F}{x_{E}},
$$

Substituindo a equação $4 \mathrm{c}$ na equação $5 \mathrm{sc}$ :

$$
k_{E}=\frac{F L}{x_{1} L_{2}+x_{2} L_{1}} .
$$

Por outro lado, da Segunda Condição de Equilíbrio (equilíbrio de rotação em torno do ponto $\mathrm{O}$, ponto de aplicação da força $\vec{F}$ - Figura 7), temos:

$$
-F_{1} L_{1}+F_{2} L_{2}=0 \rightarrow F_{1} L_{1}=F_{2} L_{2} .
$$

De (6a), $F_{2}=\left(L_{1} / L_{2}\right) F_{1}$. Substituindo em 5a),

$$
\begin{aligned}
& F_{1}\left(1+\frac{L_{1}}{L_{2}}\right)=F \rightarrow F_{1}\left(\frac{L}{L_{2}}\right)=F \\
\rightarrow & F_{1}=\left(\frac{L_{2}}{L}\right) F \rightarrow k_{1} x_{1}=\left(\frac{L_{2}}{L}\right) F .
\end{aligned}
$$




$$
x_{1}=\frac{L_{2}}{L k_{1}} F .
$$

De forma análoga,

$$
x_{2}=\frac{L_{1}}{L k_{2}} F
$$

Substituindo (6b) e (6c) na equação (5d), resulta:

$$
k_{E}=\frac{1}{\left(\frac{L_{2}}{L}\right)^{2} \frac{1}{k_{1}}+\left(\frac{L_{1}}{L}\right)^{2} \frac{1}{k_{2}}} .
$$

A equação (7) representa a predição da constante elástica equivalente $k_{E}$ na EMTAMP. Deste modelo podemse derivar as seguintes situações particulares:

- Se $L_{1}=L_{2}=L / 2$, temos:

$$
k_{E}=\frac{4 k_{1} k_{2}}{k_{1}+k_{2}} .
$$

- Se $k_{1} \approx k_{2}=k$, temos:

$$
k_{E}=\frac{k}{\left(\frac{L_{2}}{L}\right)^{2}+\left(\frac{L_{1}}{L}\right)^{2}} .
$$

- Se considerarmos tanto na equação 7.a, $k_{1} \approx k_{2}=k$, quanto na equação $7 . b, L_{1}=L_{2}=L / 2$, obtém-se:

$$
k_{E} \approx 2 k,
$$

resultando o valor que prediz o modelo tradicional (MTAMP): $k_{E}=k_{1}+k_{2} \approx 2 k[7,8,12,13]$.

Cabe destacar que, diferentemente do MTAMP, que é limitado pela hipótese de igualdade de elongações das molas associadas, a EMTAMP tem seu domínio de validade restringido pela hipótese representada na equação 4.c, que demanda que a figura formada pela haste, as molas e a barra de sustentação forme um trapézio. Na prática isso significa assumir que $\vec{x}_{1}, \vec{x}_{2}$ e $\vec{x}_{\mathrm{E}}$ são paralelos. As distâncias entre os extremos superiores e inferiores das molas são fixos, delimitados pela distância L estabelecida pelas barras que as vinculam. Se a diferença entre as elongações das duas molas $\left(x_{2}-x_{1}\right)$ é significativa em relação ao comprimento da haste $\mathrm{L}$, se perde a condição de paralelismo dos deslocamentos das extremidades inferiores das molas e a figura deixa de ser um trapézio, não sendo mais válida a hipótese sintetizada na equação 4c) (maior detalhe no material suplementar). Essa dificuldade se apresenta também quando o valor de uma das constantes de mola é maior do que o dobro do valor da outra mola, como, por exemplo, para uma situação realizada, mas não abordada neste trabalho, $\mathrm{k}_{1} \approx 4 \mathrm{k}_{2}$. Poderíamos, nesse caso, usar hastes de maior comprimento, mas, desse modo, estaríamos nos afastando da hipótese de que tais hastes possuem massa desprezível.

Para contrastar empiricamente o modelo teórico EMTAMP, compararemos os valores de $k_{E}$ estimado pelo modelo segundo a equação (7) com evidências experimentais. Para isto, coletamos dados em três situações em que as elongações das molas não são iguais: (1) para um caso em que $k_{1}$ e $k_{2}$ são semelhantes e a carga é pendurada fora do centro da haste; (2) para um caso em que $k_{1}$ e $k_{2}$ são diferentes e a carga é pendurada no mesmo local da primeira situação; e (3) para o caso em que a carga está pendurada em uma posição $L_{1}$ tal que, segundo a equação (7), a constante elástica da mola equivalente é máxima. Finalmente mostramos resultados considerando cargas suspensas em diferentes posições ao logo da haste. No que segue, apresentamos os resultados obtidos.

\subsection{Contrastação do modelo para $\mathbf{k}_{1} \approx \mathbf{k}_{2}$}

Foram coletados dados para a carga colocada mais próxima da mola 2, especificamente em $L_{1}=3 L / 4$ e $L_{2}=L / 4$, sendo o comprimento da haste $L=8 \mathrm{~cm}$. Como esperado, a mola mais próxima da carga sofre maior elongação que a mola mais distante. Da curva de ajuste aos dados experimentais, mostrados na Figura 8, obtém-se $k_{\exp }=$ $(33,2 \pm 0,9) \mathrm{N} / \mathrm{m}$ enquanto que o valor predito pela equação (7), deduzida dentro do modelo teórico EMTAMP, é $k_{E}=(31,6 \dot{s} 0,8) \mathrm{N} / \mathrm{m}$.

Considerando a incerteza da predição, constata-se que a predição e a inferência experimental se sobrepõem, o que pode ser considerado como uma evidência da validade da EMTAMP para representar o evento investigado.

\subsection{Contrastação do modelo para $\mathbf{k}_{1} \not \approx \mathbf{k}_{2}$}

Foram coletados dados em duas condições: $i$ ) com a carga pendurada no centro da haste $\left(L_{1}=L_{2}\right)$ (Figura 3.c), e ii) com a carga colocada a $3 \mathrm{~L} / 4$ da mola 1 . A configuração experimental consistiu na associação em paralelo de duas molas com constantes elásticas $k_{1}=(7,2 \pm 0,2) \mathrm{N} / \mathrm{m} e$ $k_{2}=(12,8 \pm 0,2) N / m$.

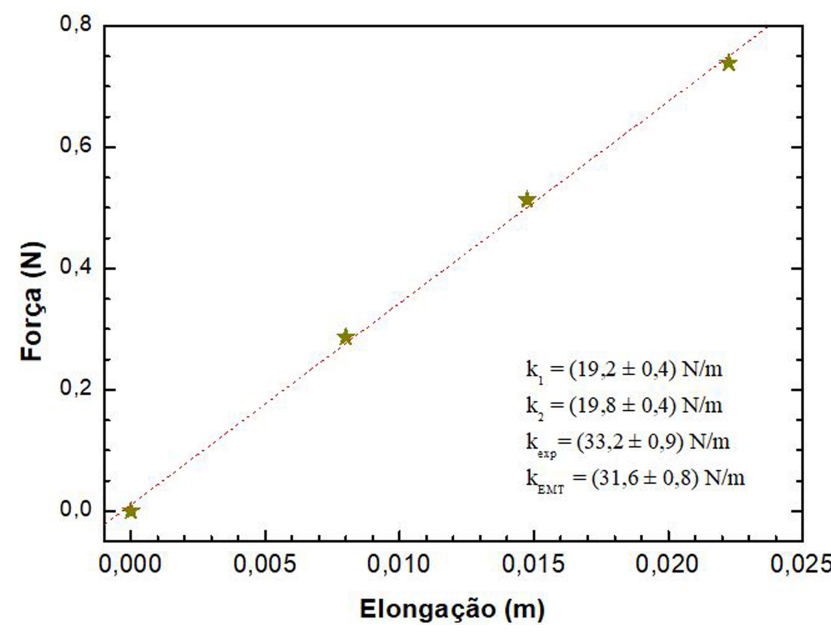

Figura 8: Força aplicada em $L_{1}=3 L / 4$ em função da elongação da mola equivalente $x_{E}$ do sistema de molas com constantes elásticas similares $\left(\mathrm{k}_{1} \approx \mathrm{k}_{2}\right)$ associadas em paralelo. $\mathrm{O}$ valor $\mathrm{da}$ constante elástica predita pelo EMTEMP é representada por $\mathrm{k}_{\mathrm{EMT}}$. 
No caso $(i)$, mesmo quando a carga é pendurada no centro da haste $\left(L_{1}=L_{2}\right)$, uma das molas sofre uma deformação maior do que a outra devido à sua menor constante de rigidez. Plotando os dados coletados da força aplicada no centro da haste em função da elongação $x_{E}$, foi feito o ajuste linear dos dados inferindo-se o valor de $k_{\text {exp }}=(18,2$ ś 0,4$) \mathrm{N} / \mathrm{m}$. Nessa configuração, a EMTAMP prediz, segundo a equação (7), o valor de $k_{E}=(18,4 \pm 0,6) \mathrm{N} / \mathrm{m}$. Novamente, considerando as incertezas relacionadas, a predição e a inferência experimental se sobrepõem, dando apoio empírico ao modelo.

No caso (ii), usando a mesma configuração experimental, coletamos dados com as mesmas duas molas, porém com a carga suspensa a 3L/4 da mola 1. Como esperado, verificamos que a mola 2 , mais próxima da carga, sofria deformação maior do que a mola 1 , mais distante. Os resultados podem ser vistos na Figura 9, onde se observa que o valor obtido para a constante de rigidez das molas associadas é $k_{\text {exp }}=(18,5 \pm 0,4) \mathrm{N} / \mathrm{m}$. Nessa configuração, a EMTAMP prediz o valor de $k_{E}=(19,0 \pm 0,7) \mathrm{N} / \mathrm{m}$. Portanto, podemos inferir que o resultado experimental corrobora empiricamente o modelo teórico contrastado.

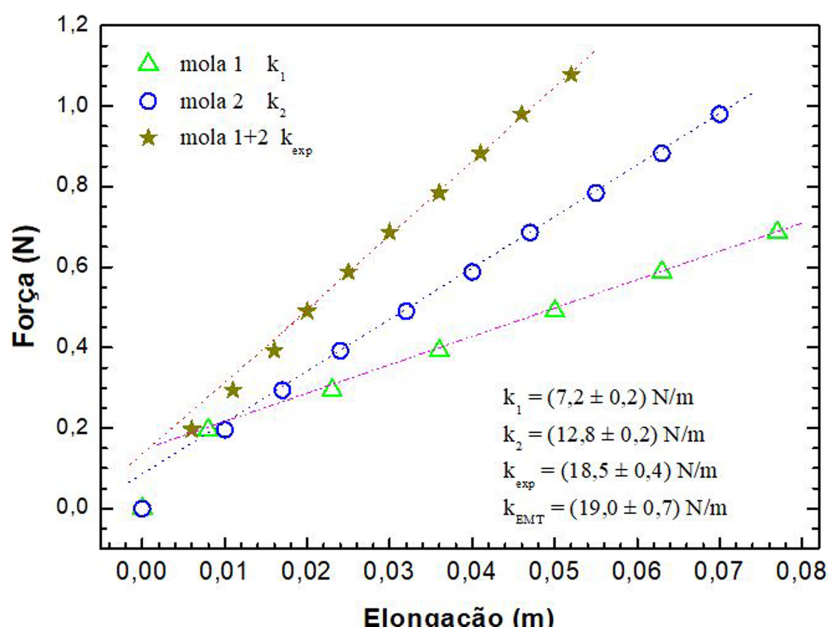

Figura 9: Força aplicada em função da elongação para as molas 1,2 (com constantes elásticas $k_{1} \not \approx k_{2}$ ) e equivalente. Nesta última, as molas 1 e 2 são associadas em paralelo, com a carga pendurada na haste a $3 \mathrm{~L} / 4$ da mola 1 . ${ }^{4} \mathrm{O}$ valor da constante elástica predito pelo EMTAMP está representado por $\mathrm{k}_{\mathrm{EMT}}$.

\footnotetext{
$\overline{{ }^{4} \text { As molas com } k_{1} \not k_{2}}$ foram elaboradas pelos autores usando arame de aço, diferentemente das molas usadas nos casos em que assumimos $k_{1} \approx k_{2}$, que eram comerciais. Ao se confeccionar as molas, observamos que elas acumulavam tensões. Quando submetidas a tratamentos térmicos, essas molas passaram a ter comportamento linear com menores elongações, mas, ainda assim, para elongações muito pequenas, elas não eram linearmente proporcionais à força aplicada, implicando que o ajuste dos dados com elas não passe na origem. Como mencionado na introdução deste artigo, esse comportamento não linear para pequenas elongações da mola foram observados e explicados por Prior [16] e Lancaster [17].
}

\subsection{Contrastação do modelo para pontos do haste onde $\mathrm{k}_{\mathrm{E}}$ é máximo.}

Da equação (7), podemos reescrever, considerando $L_{2}=$ $L-L_{1}$ :

$$
f\left(L_{1}\right)=k_{E}=\frac{1}{\left(\frac{L-L_{1}}{L}\right)^{2} \frac{1}{k_{1}}+\left(\frac{L_{1}}{L}\right)^{2} \frac{1}{k_{2}}} .
$$

O valor máximo da função $\mathrm{f}\left(L_{1}\right)$ para determinado valor de $L_{1}$ satisfaz a condição: $\frac{\partial f}{\partial L_{1}}=0$.

$\mathrm{O}$ valor de $L_{1}$ para o qual $k_{E}$ é máximo é dada por:

$$
L_{1}=\frac{k_{2} L}{k_{1}+k_{2}}
$$

Logo, de (9), substituindo os valores de $k_{1}=7,2 \mathrm{~N} / \mathrm{m}$ e $k_{2}=12,8 \mathrm{~N} / \mathrm{m}$, temos $L_{1}=0,64 \mathrm{~L}$, sendo $k_{\text {Emax }}=$ (20,0 $\pm 0,3) \mathrm{N} / \mathrm{m}$ o valor predito pelo modelo teórico a partir das equações (7) ou (8), no caso em que $L=8 \mathrm{~cm}$.

Para a contrastação empírica, foram coletados dados das elongações da associação em paralelo das duas molas para diversos valores da carga dependurada no ponto da haste em que a constante elástica equivalente é máxima $\left(L_{1}=0,64 L=5,12 \mathrm{~cm}\right)$. Tais dados constam na Tabela 1. O valor experimental para a constante elástica da associação obtido por meio do ajuste desses dados na região que apresenta comportamento linear é $k_{\text {exp }}=(19,7$ $\pm 0,2) \mathrm{N} / \mathrm{m}$. Esse dado dá suporte empírico ao modelo teórico no ponto de $k_{\mathrm{E}}$ máximo, em que a constante elástica predita tem valor (20,0 $\pm 0,3) \mathrm{N} / \mathrm{m}$.

Para estudar a validade da Eq. 7 e mostrar que a constante elástica da associação depende da posição da carga (Eq. 8), também foram determinadas as constantes elásticas da associação das duas molas, colocando a carga em diferentes pontos da haste. Os dados obtidos constam na Tabela 2 , onde são mostrados a posição da carga $\mathrm{L}_{1}$, a relação $\mathrm{L}_{1} / \mathrm{L}$, os valores de $\mathrm{k}_{\mathrm{E}}$ estimados do modelo teórico (Eq. 7) e os resultados empíricos $k_{\text {exp }}$, obtidos com as suspensão de diferentes cargas na haste para cada posição $\mathrm{L}_{1}$. Os valores teóricos contém barras de incertezas pois foram obtidos a partir dos dados experimentais (Eq. 8).

Tabela 1: Dados da elongação da associação em paralelo de duas molas em função da massa da carga colocada no ponto da haste em que $\mathrm{k}_{\mathrm{E}}$ é máximo, $\left(L_{1}=0,64 \mathrm{~L}=5,12 \mathrm{~cm}\right)$. As constantes elásticas das molas são $k_{1}=7,2 \mathrm{~N} / \mathrm{m}$ e $k_{2}=12,8$ $N / m$.

\begin{tabular}{lcc}
\hline $\mathrm{x}_{\exp }(\mathrm{m})$ & $\mathrm{m}(\mathrm{kg})$ & $\mathrm{F}=\mathrm{mg}(\mathrm{N})$ \\
\hline 0,000 & 0,000 & 0,000 \\
0,006 & 0,02005 & 0,196 \\
0,010 & 0,02995 & 0,293 \\
0,014 & 0,04005 & 0,392 \\
0,018 & 0,05005 & 0,490 \\
0,023 & 0,05995 & 0,587 \\
0,028 & 0,06990 & 0,685 \\
0,033 & 0,07995 & 0,783 \\
0,038 & 0,09005 & 0,882 \\
0,043 & 0,10015 & 0,981 \\
\hline
\end{tabular}




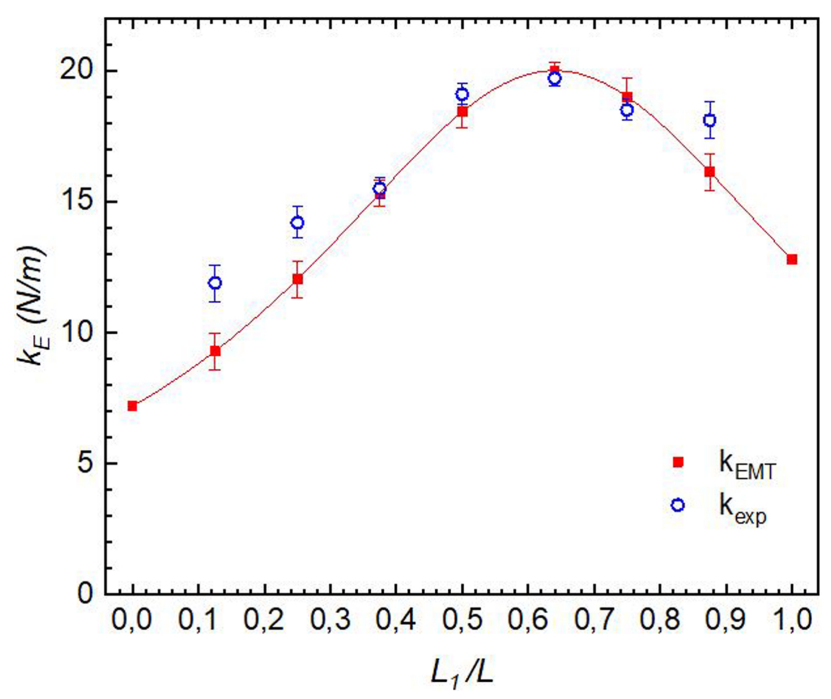

Figura 10: Constante elástica da associação de duas molas $\left(k_{1}=7,2 \mathrm{~N} / \mathrm{m}\right.$ e $k_{2}=12,8 \mathrm{~N} / \mathrm{m}$.) em paralelo em função do ponto de suspensão da carga em uma haste de $L=8 \mathrm{~cm}$, que une as molas. A curva representa os resultados previstos pelo modelo EMTEMP (Eq. 7). O valor da constante elástica predita por este modelo é representada por $\mathrm{k}_{\mathrm{EMT}}$.

Esses resultados são mostrados na Figura 10, onde se pode observar que o modelo EMTAMP descreve bem os dados experimentais no intervalo de $(0,35$ a 0,85$) L_{1} / L$. Fora dessa região, os valores previstos pelo modelo $\mathrm{k}_{\mathrm{EMT}}$ deixam de descrever com precisão os dados experimentais $\left(k_{\exp }\right)$. Isso se deve a que a hipótese de paralelismo das elongações das molas 1 e 2, assumida no modelo, deixam de ser cumpridas à medida que a carga se aproxima de uma das molas, como se pode ser visto na Figura 11.

Observe-se desta figura que o paralelismo das molas é perdido gradualmente quando afastamos a mesma carga (3 discos metálicos) da parte central $\left(\mathrm{L}_{1}=\mathrm{L} / 2\right)$ para posições próxima à mola 1 . (No material suplementar é discutida a condição de paralelismo)

Em síntese, a contrastação experimental mostra que o modelo construído levando em conta a inclinação da haste (EMTAMP) representa uma melhoria em relação

Tabela 2: Constantes elásticas experimentais para a associação de duas molas em paralelo para diferentes posição da carga, comparadas com o valor teórico (Eq. 8). As molas têm constantes elásticas $k_{1}=7,2 \mathrm{~N} / \mathrm{m}$ e $k_{2}=12,8 \mathrm{~N} / \mathrm{m}$, a haste que as une $L=8 \mathrm{~cm}$ e $L_{1}$ é a distância da mola 1 .

\begin{tabular}{lccc}
\hline $\mathrm{L}_{1}(\mathrm{~cm})$ & $\mathrm{L}_{1} / \mathrm{L}$ & $\mathrm{k}_{\exp }(\mathrm{N} / \mathrm{m})$ & $\mathrm{k}_{\text {EMT }}($ modelo $)(\mathrm{N} / \mathrm{m})$ \\
\hline $\mathbf{0 , 0 0}$ & 0,000 & - & 7,2 \\
$\mathbf{1 , 0 0}$ & 0,125 & 11,9 & 9,3 \\
$\mathbf{2 , 0 0}$ & 0,250 & 14,2 & 12,0 \\
$\mathbf{3 , 0 0}$ & 0,375 & 15,5 & 15,3 \\
$\mathbf{4 , 0 0}$ & 0,500 & 19,1 & 18,4 \\
$\mathbf{5 , 1 2}$ & 0,640 & 19,7 & 20,0 \\
$\mathbf{6 , 0 0}$ & 0,750 & 18,5 & 19,0 \\
$\mathbf{7 , 0 0}$ & 0,875 & 18,1 & 16,1 \\
$\mathbf{8 , 0 0}$ & 1,000 & - & 12,8 \\
\hline
\end{tabular}

ao modelo tradicional, contudo, como esperado, pois os modelos científicos são representações da realidade, o modelo expandido também possui um domínio de validade limitado.

\section{Comentários finais}

Um costume dos livros de ensino é de fazer analogias entre situações físicas diferentes, explorando algumas de suas representações, sejam visuais, algébricas e ou ideias que apresentarem similaridade. Em termos gerais, uma analogia é, frequentemente, entendida como a comparação baseada em similaridades entre estruturas de dois domínios de conhecimento diferentes, um mais familiar (denominado "análogo") e outro desconhecido ou menos conhecido (denominado "alvo") [26]. Por outro lado, Fonseca e Trindade [27] expõe a importância das analogias ao se referirem ao estudo de Einstein da teoria quântica do gás ideal monoatómico, mais conhecida como Condensação de Bose-Einstein. Einstein usou como analogia o estudo de uma condensação em gases reais, não obstante a condensação que estudava ter origem física distinta.

Em relação à associação de molas, em particular, é comum nos livros didáticos se fazer analogia entre a associação de molas e a associação de resistores, o que pode levar a raciocínios equivocados. Por exemplo, na associação em paralelo de molas no modelo tradicional, a constante elástica equivalente é dada como a soma $k_{E}=$ $k_{1}+k_{2}$. Já no caso do modelo de resistores, a resistência equivalente é dada por $\frac{1}{R_{E}}=\frac{1}{R_{1}}+\frac{1}{R_{2}}$. Uma analogia mais coerente seria entre a associação de molas e a associação de capacitores, em que ambos os modelos apresentam estrutura algébrica similar. No caso da associação em paralelo de dois capacitores, a capacitância equivalente é dada por $C_{E}=C_{1}+C_{2}$. Agora, se entramos nos pormenores dos modelos, veremos que mesmo sendo as estruturas algébricas similares, as ideias inseridas nos modelos não se correspondem. Por exemplo, no sistema de dois capacitores associados em paralelo a diferença de potencial elétrico em ambos os capacitores é a mesma. No entanto, não necessariamente a força F, a grandeza correspondente à diferença de potencial para a associação de molas, é a mesma para as duas molas associadas, pois essa força é distribuída na haste. A hipótese no modelo
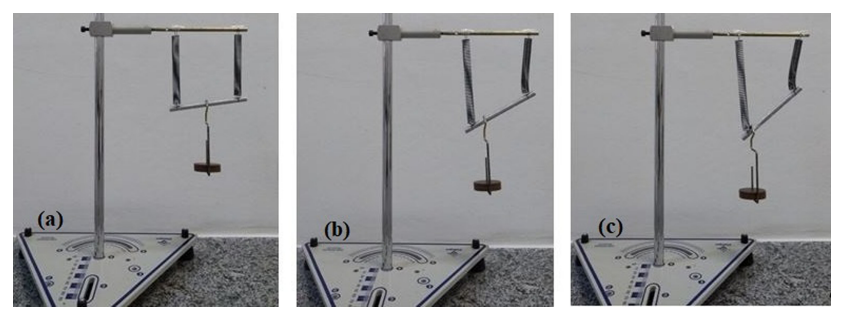

Figura 11: A condição de paralelismo das molas é perdida gradualmente à medida que a mesma carga se afasta $(a)$ do centro da mola $\mathrm{L}_{1}=\mathrm{L} / 2$, para $(b) \mathrm{L}_{1}=2 \mathrm{~L} / 8$ e $(c) \mathrm{L}_{1}=\mathrm{L} / 8$. 
da associação de capacitores é que, no equilíbrio, a carga equivalente é dada por $q_{E}=q_{1}+q_{2}$, enquanto o análogo da elongação equivalente é dada por $x_{E}=x_{1}=x_{2}$. Ou seja, a rigor não basta que a estrutura algébrica seja similar para se ter uma analogia adequada.

Como diria um dos árbitros deste artigo, no caso de circuitos elétricos "a equivalência tem um aspecto absoluto", pois, por exemplo, na associação de resistores os diversos parâmetros (corrente total, tensão fornecida pela fonte de diferença de potencial e dissipação total de energia através do efeito Joule) são os mesmos, tanto no circuito original como no equivalente. Já no caso da associação de molas "a equivalência tem um aspecto relativo", pois $k_{E}$ e $x_{\mathrm{E}}$ dependem de características intrínsecas $\left(L_{1}\right.$ e $\left.L_{2}\right)$ e extrínsecas do sistema $\left(F_{1}\right.$ e $\left.F_{2}\right)$. O produto $k_{\mathrm{E}} \cdot x_{\mathrm{E}}$ se ajusta para que $F_{\mathrm{E}}$ não dependa de tais características.

Uma analogia interessante entre um circuito hidráulico (domínio familiar) e o circuito elétrico (domínio alvo) encontra várias correspondências interessantes, como: A bomba faz circular o fluido no circuito hidráulico com uma diferença de pressão enquanto, a bateria faz circular as cargas elétricas no circuito elétrico com uma diferença de potencial elétrico, entre outras considerações [27]. Porém, também podem levar a equívocos, como o aluno pensar que a velocidade de movimento das cargas elétricas é igual à velocidade de transmissão da energia elétrica.

No presente estudo, é ousado fazer analogia do modelo proposto da associação de molas em paralelo com a associação de resistores ou de capacitores em circuitos elétricos. Uma possibilidade de analogia seria com circuitos hidráulicos em paralelo. Para isso, seria necessária uma análise criteriosa, o que foge do escopo deste artigo.

Ante isso, todo cuidado é pouco com as analogias que se fazem na física ou na ciência em geral, como mesmo Bunge ensina [1974, p. 199]:

Em resumo, a analogia é indubitavelmente fecunda, mas dá nascimento tanto a monstros como a bebês sadios. E, em qualquer dos casos, seus produtos, assim como os da intuição, são apenas isto: recém-nascidos que precisam ser criados, se é que o precisam, mais do que cultuados. Em outros termos, encontrar uma analogia (i.é, construir um argumento que contenha juízos de analogia) é apenas um começo.

O entendimento de que o enfoque na modelagem científica é promissor para a realização de debates sobre filosofia da Ciência nas salas de aula permeia a literatura da área de ensino de Física [4-7]. No entanto, concretizar esses debates em suas aulas pode ser um grande desafio para professores de Física.

Neste artigo, buscando contribuir com um exemplo para a promoção de debates sobre o processo de modelagem científica no ensino de Física, apresentamos uma situação em que a compreensão do processo de construção de modelos teóricos é fundamental para uma conscientização das possibilidades e das limitações do conhecimento científico. Particularmente, analisamos o domínio de validade de dois modelos que representam a associação de duas molas em paralelo, mas que se fundamentam em distintas simplificações da realidade. O primeiro dos modelos discutidos costuma ser abordado em livros didáticos de Física, mas frequentemente desacompanhado de reflexões sobre os seus pressupostos básicos, como o pressuposto de que as duas molas associadas em paralelo se distendem igualmente. Neste artigo, evidenciamos as idealizações consideradas nesse modelo, discutimos a influência delas nas predições construídas, e o contrastamos experimentalmente, mostrando que o seu domínio de validade abarca a situação em que duas molas de constantes elásticas semelhantes são associadas.

Destacamos então que, em eventos reais, dificilmente teremos elongações semelhantes quando associamos duas molas em paralelo. Por isso, o segundo modelo discutido neste artigo é uma expansão do modelo tradicionalmente abordado em livros didáticos. Construímos esse modelo nos amparando na idealização de que as molas associadas permanecem perfeitamente paralelas quando são distendidas, ainda que essas elongações possam ser diferentes para cada uma das duas molas associadas. Realizamos a sua contrastação empírica em três situações: $i$. para um caso em que as constantes elásticas das molas associadas são semelhantes, mas a força que as elonga é aplicada fora do centro da haste que as une; ii. para um caso em que as constantes elásticas das molas são diferentes e a força que elonga as molas é aplicada no mesmo local da primeira situação; e iii. para o caso em que a força sobre a haste que une as molas é aplicada em uma posição tal que a constante elástica da mola equivalente é a máxima possível. A comparação entre as predições construídas e os dados coletados experimentalmente mostraram que essas três situações estão dentro do domínio de validade do modelo teórico expandido.

A partir da discussão realizada sobre as implicações das simplificações da realidade consideradas nesses dois modelos teóricos sobre a associação de duas molas em paralelo, evidenciamos o caráter representacional dos modelos científicos, mostrando que a construção deles envolve intuição e razão durante o estabelecimento das idealizações consideradas, e que eles são suscetíveis de serem melhorados segundo as necessidades do investigador [10].

Além disso, procuramos destacar a importância das atividades experimentais no processo de contrastação das ideias científicas como parte importante no processo de formação inicial como investigador. Na contrastação empírica realizada, enfatizamos o processo de controle de variáveis pautado pelas simplificações da realidade consideradas no conhecimento científico contrastado, que é intrínseco ao fazer experimental, oferecendo uma interpretação alternativa sobre o papel do trabalho experimental que se afasta de concepções empiristas-indutivistas ingê- 
nuas. Expomos a experimentação como uma atividade focada na avaliação das implicações das idealizações e aproximações assumidas nos modelos científicos nos seus domínios de validade e graus de precisão.

Desse modo, pretendemos contribuir para a área de ensino tanto para enriquecer as reflexões sobre como a natureza da Ciência pode ser implementada em aulas de Física por meio da experimentação, como para proporcionar uma discussão sobre um modelo teórico da Física que é insuficientemente discutido em livros didáticos.

\section{Agradecimentos}

Agradecemos ao professor Ives Solano Araujo pelo incentivo e sugestões ao trabalho e, especialmente, aos árbitros pela leitura crítica e observações que contribuíram para a melhora do presente trabalho.

\section{Material Suplementar}

O seguinte material suplementar está disponível online:

Paralelismo das molas na associação de molas em paralelo

\section{Referências}

[1] L.A. Heidemann, I.S. Araujo e E.A. Veit, Caderno Brasileiro de Ensino de Física 33, 3 (2016).

[2] R.V. Brandão, I.S. Araujo e E.A. Veit, Caderno Brasileiro de Ensino de Física 28, 507 (2011).

[3] A. Raviolo, P. Ramírez e E. López, Revista Eureka sobre Enseñanza y Divulgación de las Ciencias 7, 581 (2010).

[4] M. Pietrocola, Investigações em Ensino de Ciências 4, 213 (1999).

[5] .J. Jackson, L. Dukerich e D. Hestenes, Science Educator 17, 10 (2008).

[6] J. Gilbert, International Journal of Science and Mathematics Education 2, 115 (2004).

[7] I.T. Koponen, Science\&Education 16, 751 (2007).

[8] A. Cupani e M. Pietrocola, Caderno Brasileiro de Ensino de Física 19, 100 (2002).

[9] J. Machado e M. Braga, Caderno Brasileiro de Ensino de Física 36, 178 (2019).

[10] M. Bunge, Teoria e Realidade (Perspectiva, São Paulo, 1974).

[11] P.A. Atam, Introduction to Classical Mechanics (Upper Sadle River: Prentice Hall, 1998), $2^{\text {a }}$ ed., p. 108.

[12] H.D. Young e R.A. Freedman, Física I: Mecânica (Addison Wesley, São Paulo, 2008), 12a ed., p. 207.

[13] A.E. Moyer, Isis 6, 242 (1977)

[14] L.U. Mutzenberg, E.A. Veit e F.L. da Silveira, Revista Brasileira de Ensino de Física 26, 307 (2004).

[15] N. Aranha, J.M. de Oliveira Jr, L.O. Bellio e W. Bonventi Jr, Revista Brasileira de Ensino de Física 38, 4305 (2016).

[16] R.M. Prior, Phys. Teach. 18, 601 (1980).

[17] G. Lancaster, Phys. Educ. 18, 217 (1983).

[18] R. Schwartz e B. Skjold, Educación Química, 23, 451 (2012).
[19] D. Treagust, G. Chittleborough e T.L. Mamiala, International Journal of Science Education 24, 357 (2002).

[20] J. Torres e C. Vasconcelos, Eurasia Journal of Mathematics, Science \& Technology Education 11, 1473 (2015).

[21] J.D. Serna e A. Joshi, Physics Education 46, 33 (2011).

[22] P. Gluck, Physics Education 45, 178 (2010).

[23] M. Nussenzveig, Curso de Física Básica: Mecânica - Vol. 1 (Editora Blucher, São Paulo, 2013), $5^{\mathrm{a}}$ ed.

[24] J. Peruzzo, A Física Através de Experimentos: Mecânica (Editora Irani/SC, 2013), $1^{\mathrm{a}}$ ed., p. 72.

[25] M.M. Lucchese, G.F. Marranghello e F.S. da Rocha, Física na Escola 16, 22 (2018).

[26] M.C. Duarte, Investigações em Ensino de Ciências 10, 7 (2005).

[27] J. Fonseca e Trindade, em Atas do XI Congresso da Sociedade Portuguesa de Ciências da Educação (SPCE, Guarda, 2011), disponível em https://www researchgate.net/publication/234004642, acessado em 30/08/2019. 